\title{
Funding should recognize the value of peer review
}

\section{This service to science is threatened by time constraints and performance assessment.}

Sir - The peer-review system, discussed in your News feature "Publish, and be damned..." (Nature 419, 772-776; 2002), operates almost entirely on a voluntary basis. It carries some prestige, but does not really affect an individual's academic progress. This is wrong, for two reasons.

First, to whichever end of the "scientific-publishing food chain" (as your feature engagingly calls it) it is applied, peer review plays a major role in preventing pollution of scientific data with falsified or distorted information. It also decreases wasteful publication of bad science.

Second, it introduces an element of additional, independent judgment, which eliminates noise and clarifies interpretation. Thus, like an audit in the medical world, peer review is crucial in ensuring that science works properly.

In spite of all this, peer review is becoming more difficult to apply because of the increasingly scarce commodity of time. A substantial number of potential referees are declining to accept journals' invitations to review, or are unable to deliver a report, because of time constraints.

Thus, there must be more institutional support of peer review. Although scientists and editors recognize that being invited to review is an act of academic recognition, assessment-conscious institutions fail to recognize this. Yet peer review is an important service to science, and it seems obvious that scientific institutions should support it very strongly. A ranking system is needed, reflecting an individual's participation in the peer-review process, similar to his or her publication record, and this should be taken into account in performance assessments. The system could be weighted according to the impact factors of the journals served, and there could be an element of funding associated with an institution's peer-review score.

All this is necessary to take into account ever-increasing time pressures that could affect the quality of science. Peer review does not need to change fundamentally, but it should be moved higher up the list of academic priorities.

Marek H. Dominiczak

Medical Humanities Unit, Gartnavel General

Hospital, 1053 Great Western Road,

Glasgow G12 OYN, UK

\section{Biology can be helpful to open-minded physicists}

Sir - Your News Feature "Bridging the culture gap" is a welcome update on the status of interdisciplinary research in biology, as relevant today as it was 15 years ago when Arthur Kornberg commented on a similar topic ${ }^{2}$.

Communication is a major barrier that impedes collaboration between biologists and physicists.

Biologists tend to start by asking a specific question for a specific biological system. The answers to specific questions gradually contribute to the delineation of general principles.

Many physicists, when addressing biological problems, consider themselves as generalists who are more interested in developing general theories or finding evidence to fit them.

Although physicists can dismiss biologists' explanations as too "empirical and descriptive", this level of explanation is apparently good enough for biologists and has served biology well in practice. Physicists' quantitative explanations may well provide a deeper level of mechanistic understanding, but deeper is not necessarily better if important biological context is lost. The satisfaction of scientific explanation is a relative term and exists in the eye of the beholder. Insisting on the most fundamental explanation for every biological phenomenon brings us all the way down to the elementary particles, which is beyond the scope not only of biology but also of many subdisciplines in physics.
The physicists who make the greatest strides in interdisciplinary research are open-minded, genuinely interested in biological problems and determined to make the transition. They apply quantitative techniques to address questions of interest to biologists.

John Hopfield is an excellent example of a physicist whose theoretical models have always focused on biological functions. In addition to his work on neural networks in the 1980s, as mentioned in your feature, in the 1970 s Hopfield developed a series of elegant models on kinetic proofreading to explain the extremely low error rate in the biosynthesis of macromolecules and kinetic cooperativity of haemoglobin (see, for example, refs 3 and 4 respectively). These models and their variations have been enthusiastically embraced by biologists and are now being applied to areas of immunology, signal transduction, intracellular transport, DNA disentanglement and protein folding in vivo to explain why small quantitative changes in molecular interactions can lead to qualitative differences in biological function, as well as how biochemical energy can be used to improve the accuracy of molecular processes.

Although the idea of interdisciplinary research has been around for a long time and has been actively promoted by agencies such as the US National Science Foundation and Department of Energy, it is far from reaching its potential.

It is to be hoped that opportunities in genomics and other data-rich biological fields can finally provide the necessary attraction to drive more and more interested physicists towards biologyand to keep them there.

\section{Guangpu Li}

Department of Biochemistry and Molecular Biology, University of Oklahoma Health Sciences Center, 940 S.L. Young Boulevard, BMSB 853, Oklahoma City, Oklahoma 73104, USA

1. Knight, J. Nature 419, 244-246 (2002).

2. Kornberg, A. Biochem. 26, 6888-6891 (1987).

3. Hopfield, J. J. Proc. Natl Acad. Sci. USA 77, 5248-5252 (1980).

4. Hopfield, J. J., Shulman, R. G. \& Ogawa, S. J. Mol. Biol. 61, 425-433 (1971).

\section{Strict guidelines make it clear who's responsible}

Sir — The headline "Physics guidelines drop equal-responsibility clause", on your News story (Nature 420, 258; 2002) reporting new ethical guidelines adopted by the American Physical Society (APS) in response to recent cases of misconduct, leaves the impression that the APS weakened its guidelines. On the contrary, the guidelines have been strengthened.

A major reason for the APS revision was to make explicit the responsibilities of co-authors. In particular, the new version includes a provision that specifies some authors who must take full responsibility for the content of an entire paper.

As your News story indicates, some physicists feel that the guidelines are now too strict, but most of them are supportive. The APS will continue to consider its role in establishing ethical guidelines and promoting education in professional conduct.

Myriam P. Sarachik

President, American Physical Society, 1 Physics Ellipse, College Park, Maryland 20740-3844, USA 